\title{
Adaptive Envelope Shaping for Low and Medium Power Amplifiers with Dynamic Supply
}

\author{
Pere L. Gilabert, Senior Member, IEEE, Gabriel Montoro, Member, IEEE, Nieves Ruiz, Student Member, IEEE \\ and José A. García, Member, IEEE
}

\begin{abstract}
This paper presents an adaptive envelope shaping (AES) method to linearize low and medium power amplifiers (PAs) with dynamic supply. This straightforward linearization solution results very useful for small cell or handset transmitters, where reducing power consumption and computational complexity of the digital part is crucial. With the AES method, there is no need of an a priori characterization of the PA to shape the supply voltage signal targeting maximum linearity. Excellent linearization results are obtained when the PA presents good AMPM linearity, otherwise, additional phase distortion linearization has to be included to meet the ACLR specifications.
\end{abstract}

Index Terms-Envelope shaping, linearization, power amplifier.

\section{INTRODUCTION}

$\mathbf{E}$ NVELOPE tracking (ET) and envelope elimination and restoration (EER) are supply modulation techniques suitable to complement existing fixed supply transmitter architectures by enhancing the power amplifier (PA) power added efficiency (PAE), critically degraded when using multicarrier and spectrally efficient modulation schemes presenting high peak to average power ratio (PAPR). The overall power efficiency in ET and EER not only depends on both RF PA and envelope amplifier (EA), but also on the computational load introduced by the linearization algorithm, since the power consumption of the digital part is dominant in small cell transmitters.

By using an envelope shaping function [1], the instantaneous supply voltage can be chosen to either achieve optimum efficiency at the cost of having nonlinear distortion at the PA output or, alternatively, to achieve certain levels of linearity at the cost of a small loss of efficiency (e.g., Nujira-Wilson or $\mathrm{N}=6$ shaping in [2]). To minimize the impact of the linearization subsystem (in terms of computational complexity and energy efficiency) in pico/femto cells or handsets, the envelope shaping function aims to compensate for the PA's AMAM distortion. This linearization method assumes the AMPM distortion to be negligible, otherwise, additional phase distortion compensation (e.g., [3]) has to be included in the in-phase (I) and quadrature-phase (Q) path.

Manuscript received July 31, 2015; revised March 2, 2016; accepted April 12, 2016. This work was partially supported by the Spanish Government (MINECO) and FEDER under projects TEC2014-58341-C4-01-R and 03-R; by the Catalan Government under project 2014-SGR-1103; and by CATRENE under the project named CORTIF CA116.

P. L. Gilabert and G. Montoro are with the Dept. of Signal Theory and Communications, Universitat Politècnica de Catalunya (UPC) - Barcelona Tech. C/ Esteve Terradas, 7, 08860 Castelldefels, Spain. (+34 934137 211; e-mail: plgilabert@tsc.upc.edu, gabriel.montoro@upc.edu).

N. Ruiz and J. A. García are with the Dept. of Communications Eng., Universidad de Cantabria (UC) - Plaza de la Ciencia s/n, 39005 Santander, Spain. (e-mail: mariadelasnieves.ruiz@unican.es, joseangel.garcia@unican.es).
To design the envelope shaping function, a previous characterization of the PA is necessary (e.g., gain versus output power curves for different supply voltages). Normally, this characterization is carried out through $\mathrm{CW}$ test signals. However, this measurements are not always feasible or reliable, since thermal and bias network equilibrium are different for $\mathrm{CW}$ versus modulated signals. For example, PAs based on GaN HEMT transistors experience a soft-compression effect when the AM-AM measurement is made under static conditions [4]. Moreover, certain topologies of the EA do not allow the use of CW excitation due to its small PAPR and the need of stressing the PA with a high mean output power in order to characterize the peaking range. As a consequence, it is best if the extraction of the different gain characteristic curves are done under a dynamic excitation (e.g., LTE waveforms). This ultimately leads to a lack of precision of the characteristic curves (i.e., blurring of the measured data) that impacts the linearization performance of the envelope shaping function (e.g., unbalanced ACLR compensation).

This letter presents an adaptive envelope shaping (AES) method aimed at compensating for the AM-AM distortion that avoids the need of an a priori characterization of the PA (i.e., generating an envelope shaping function by interpolating a set of unprecise measurements). Moreover, unlike Nujira's isogain shaping described in [2], thanks to its closed-loop nature it can be used for operating in both ET and EER modes and it does not need to be pre-adjusted.

\section{Adaptive EnVelope Shaping}

The block diagram of a power efficient transmitter with dynamic supply, including the proposed adaptive envelope shaping (AES) subsystem, is depicted in Fig. 1. Considering the baseband complex signal to be transmitted $u[n]$, the envelope is defined as $E[n]=\sqrt{u_{I}^{2}[n]+u_{Q}^{2}[n]}$. The inputoutput relationship of the envelope shaping function (SF) is defined (see Fig. 1) as

$$
E_{s}[n]=f_{\mathrm{sF}}(E[n])=E[n]-\varepsilon[n]
$$

where $\varepsilon[n]$ is the nonlinear distortion signal that, taking also into account memory terms, can be modeled as

$$
\varepsilon[n]=\sum_{i=0}^{N-1} \sum_{p=0}^{P-1} w_{p, i} \cdot\left(E\left[n-\tau_{i}\right]\right)^{p}=\varphi_{n} \mathbf{w}_{n}
$$

where $\tau_{i}$ (with $\tau \in \mathbb{Z}$ and $\tau_{0}=0$ ) are the most significant sparse delays of the envelope. In matrix notation, 


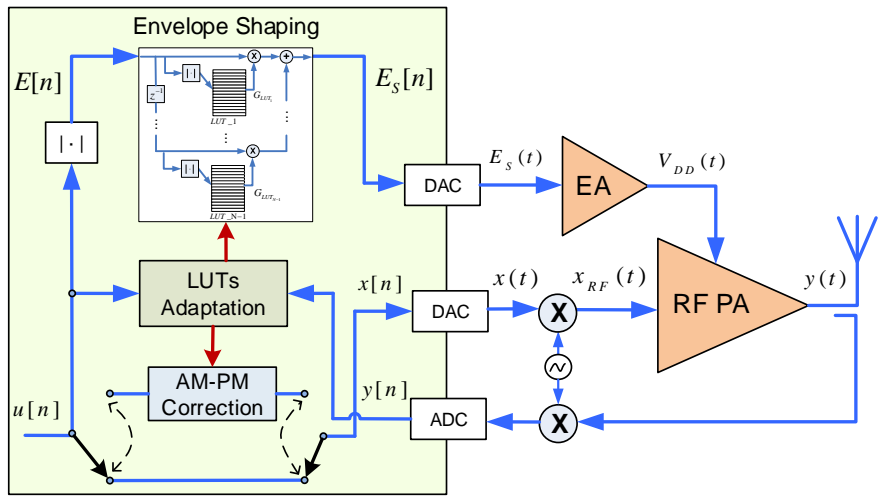

Fig. 1. Block diagram of the PA with dynamic supply including adaptive envelope shaping and optional I-Q DPD.

$\mathbf{w}_{n}=\left(w_{0,0}, \cdots, w_{P-1,0}, \cdots, w_{0, N-1} \cdots, w_{P-1, N-1}\right)^{T}$ is a vector of coefficients with dimensions $O x 1$, where $O=$ $P \cdot N$, is the order of the behavioral model in (2), $P$ is the polynomial order and $N$ the number of delays; and $\varphi_{n}=$ $\left(E[n], \cdots,(E[n])^{p}, \cdots, E\left[n-\tau_{N-1}\right], \cdots,\left(E\left[n-\tau_{N-1}\right]\right)^{p}\right)$ is the $1 x O$ data vector containing the basis waveforms. Following a closed-loop error minimization technique, the coefficients in (2) can be extracted iteratively using a weighted least squares algorithm,

$$
\mathbf{w}_{n+1}=\mathbf{w}_{n}+\mu\left(\boldsymbol{\Phi}^{\mathbf{H}} \boldsymbol{\Phi}\right)^{-1} \boldsymbol{\Phi}^{\mathbf{H}} \mathbf{e}
$$

with $\mathbf{\Phi}=\left(\boldsymbol{\varphi}_{0}, \boldsymbol{\varphi}_{1} \cdots, \boldsymbol{\varphi}_{L}\right)^{T}$ being the $L x O$ data matrix, where $L$ is the number of data samples $(n=1,2, \cdots L)$, and $\mu(0 \leq \mu \leq 1)$ being the weighting factor. Finally, $\mathbf{e}$ is the $L x 1$ vector of the error defined as

$$
\mathbf{e}=\left|\frac{\mathbf{y}}{G_{0}}\right|-\mathbf{E}
$$

where $G_{0}$ determines the desired linear gain of the PA, and where $\mathbf{y}$ and $\mathbf{E}$ are the $L x 1$ vectors of the PA output and baseband instantaneous envelope, respectively.

Targeting a future FPGA implementation, the AES can be easily carried out using LUTs. Therefore, (1) and (2) can be rewritten as the combination of $N$ LUTs,

$$
E_{s}[n]=E[n]-\sum_{i=0}^{N-1} E\left[n-\tau_{i}\right] \cdot G_{L U T_{i}}\left(E\left[n-\tau_{i}\right]\right)+w_{0 i}
$$

where $w_{0 i}$ are the offset coefficients that have a big impact on the linearity vs. efficiency trade-off [5].

\section{EXPERIMENTAL SETUP AND RESUlTS}

The experimental test-bench is depicted in Fig. 2. For testing purposes, we used a broadband high efficiency continuousmode class-J power amplifier at $950 \mathrm{MHz}$, based on the CGH35030F packaged GaN HEMT from Cree Inc. The signal generation and measurement equipment consist of: Texas Instruments boards (TSW1400EVM pattern generator + TSW30H84EVM DACs and I-Q modulator), a Tabor WW2572A arbitrary wave generator, and a Keysight Infinium DSO9404A oscilloscope for capturing the RF signals. Another oscilloscope is used for capturing the drain voltage and current,

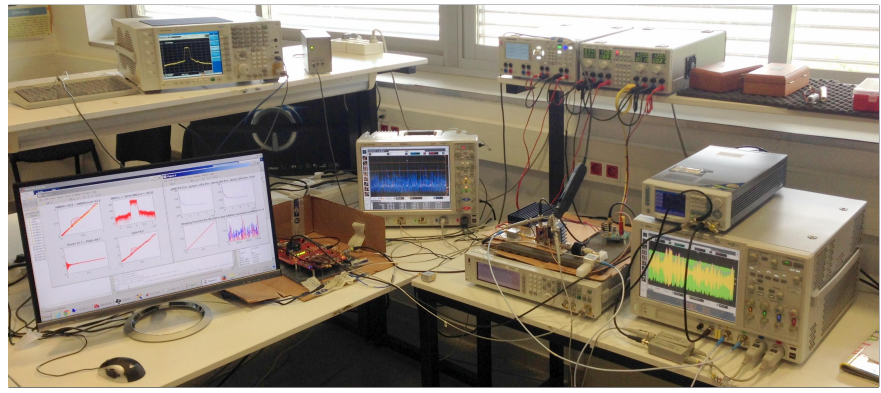

Fig. 2. Experimental test bench.

and this information is used for calculating the drain power consumption. A PC running Matlab controls all the instrumentation and does all the required digital signal processing. We used as the EA the high-speed ( $35 \mathrm{MHz}$ bandwidth and 900 $V / \mu s$ slew-rate at $\mathrm{Av}=2$ and $10 \Omega$ load) high-current (1.1 A) Linear Technology IC LT1210. For the sake of simplicity we considered the linear but slightly efficient IC LT1210 as the EA. Thus, the drain biasing voltage could be lowered down to $0 \mathrm{~V}$ while the reported PAE values take only into account the consumption at the RF PA drain. The scope of this work is to prove the linearity performance of the proposed AES method. The signal used was an uplink LTE signal of $5 \mathrm{MHz}$ bandwidth and $8.3 \mathrm{~dB}$ of PAPR. The targeted ACLR levels to meet the specifications for the LTE uplink channel were $-38 \mathrm{~dB}$. Table I shows the ACLR, NMSE and PA's PAE when using the proposed AES method for different mean output power levels and dynamic supply strategies, namely, ET and EER. To detect the importance of the AM-PM distortion, and thus determine the linearization limits of the AES method, we can compare the NMSE of the modulus (where the error is defined as in (4)) with the classical NMSE for complex signals. This latter error is defined as $\mathbf{e}=\left|\frac{\mathbf{y}}{G_{0}}-\mathbf{u}\right|$, with $\mathbf{u}$ and $\mathbf{y}$ being the RF PA input and output signals (see Fig 1), respectively. If the PA shows significant AM-PM distortion (evidenced in the $\triangle$ NMSE column in Table I), with only AES linearization may not be enough to meet the ACLR specifications, and additional phase distortion compensation may be required in the I-Q path. However, no mutual optimization of the inputs (supply voltage and RF input amplitude) is done. Fig. 3 to Fig. 6 show the AMAM, AM-PM and output power spectra for both ET and EER dynamic supply strategies when considering: no shaping (i.e., $E_{s}[n]=E[n]$ in (1)), memoryless AES and, AES + Phase DPD (in the case of ET) or AES with memory compensation - 4 LUTs - (in the case of EER, to compensate for the dynamic dependency on the supply voltage).

\section{CONCLUSION}

The proposed AES method is capable of compensating for the AM-AM distortion of PAs with dynamic supply with a single LUT and without any a priori characterization of the PA. Experimental results showed that operating in ET mode additional phase distortion compensation was necessary to meet the - $38 \mathrm{~dB}$ ACLR specifications. Instead, in EER mode, with the class-J PA driven deep into saturation (see the PA gain column in Table I) and operating close to a class-E switched- 
TABLE I

COMPARISON OF Linearization STRATEgIES FOR LOW AND MEDIUM POWER AMPLIFIERS With DyNAMIC SUPPLY.

\begin{tabular}{|c|c|c|c|c|c|c|c|c|c|c|}
\hline & \multirow{2}{*}{$\begin{array}{l}\text { Linearization } \\
\text { Scheme }\end{array}$} & \multirow{2}{*}{$\begin{array}{c}\mathrm{P}_{\text {out }} \\
(\mathrm{dBm})\end{array}$} & \multirow{2}{*}{$\begin{array}{l}\text { PA Gain } \\
\text { (dB) }\end{array}$} & \multirow{2}{*}{$\begin{array}{l}\text { Supp. Voltage } \\
\text { Swing (V) }\end{array}$} & \multicolumn{2}{|c|}{ ACLR (dB) } & \multirow{2}{*}{$\begin{array}{c}\text { NMSE } \\
(\mathrm{dB})\end{array}$} & \multirow{2}{*}{$\begin{array}{c}\text { NMSE } \\
\text { Modulus (dB) }\end{array}$} & \multirow{2}{*}{$\begin{array}{c}\Delta \text { NMSE } \\
(\mathrm{dB})\end{array}$} & \multirow{2}{*}{$\begin{array}{l}\text { PA PAE } \\
(\%)\end{array}$} \\
\hline & & & & & Upper & Lower & & & & \\
\hline \multirow{6}{*}{ ET } & No Shaping & \multirow{4}{*}{27.0} & \multirow{4}{*}{16.3} & \multirow{4}{*}{$\begin{array}{c}13-20 \\
\left(\mathrm{v}_{\mathrm{GS}}=-3.4 \mathrm{~V}\right)\end{array}$} & -23.1 & -24.7 & -15.2 & -15.6 & 0.4 & 39.3 \\
\hline & Memoryless AES (1-LUT) & & & & -31.9 & -31.9 & -24.1 & -36.0 & 11.9 & 33.3 \\
\hline & AES + Memory (4-LUTs) & & & & -32.1 & -30.0 & -22.9 & -32.6 & 9.7 & 33.1 \\
\hline & AES (1-LUT) + Phase DPD & & & & -45.4 & -46.2 & -32.5 & -35.5 & 3.0 & 33.1 \\
\hline & Memoryless AES (1-LUT) & \multirow{2}{*}{31.5} & \multirow{2}{*}{18.0} & $19-28$ & -29.7 & -33.1 & -22.2 & -36.4 & 14.2 & 47.2 \\
\hline & AES (1-LUT) + Phase DPD & & & $\left(\mathrm{v}_{\mathrm{GS}}=-3.4 \mathrm{~V}\right)$ & -42.4 & -43.4 & -33.2 & -35.8 & 2.6 & 46.5 \\
\hline \multirow{6}{*}{ EER } & No Shaping & \multirow{4}{*}{27.1} & \multirow{4}{*}{7.0} & \multirow{4}{*}{$\begin{array}{c}0-20 \\
\left(\mathrm{v}_{\mathrm{GS}}=-3.4 \mathrm{~V}\right)\end{array}$} & -33.6 & -37.3 & -27.3 & -30.1 & 2.8 & 40.9 \\
\hline & Memoryless AES (1-LUT) & & & & -38.0 & -40.1 & -30.2 & -39.0 & 8.8 & 40.5 \\
\hline & AES + Memory (4-LUTs) & & & & -39.8 & -40.3 & -31.3 & -41.7 & 10.4 & 39.5 \\
\hline & AES (1-LUT) + Phase DPD & & & & -43.0 & -44.5 & -36.1 & -38.8 & 2.7 & 41.4 \\
\hline & Memoryless AES (1-LUT) & \multirow{2}{*}{31.1} & \multirow{2}{*}{8.1} & $0-28$ & -43.0 & -41.6 & -35.3 & -39.2 & 3.9 & 49.0 \\
\hline & AES + Memory (4-LUTs) & & & $\left(\mathrm{V}_{\mathrm{GS}}=-3.4 \mathrm{~V}\right)$ & -44.0 & -43.6 & -36.3 & -42.1 & 5.8 & 49.0 \\
\hline
\end{tabular}
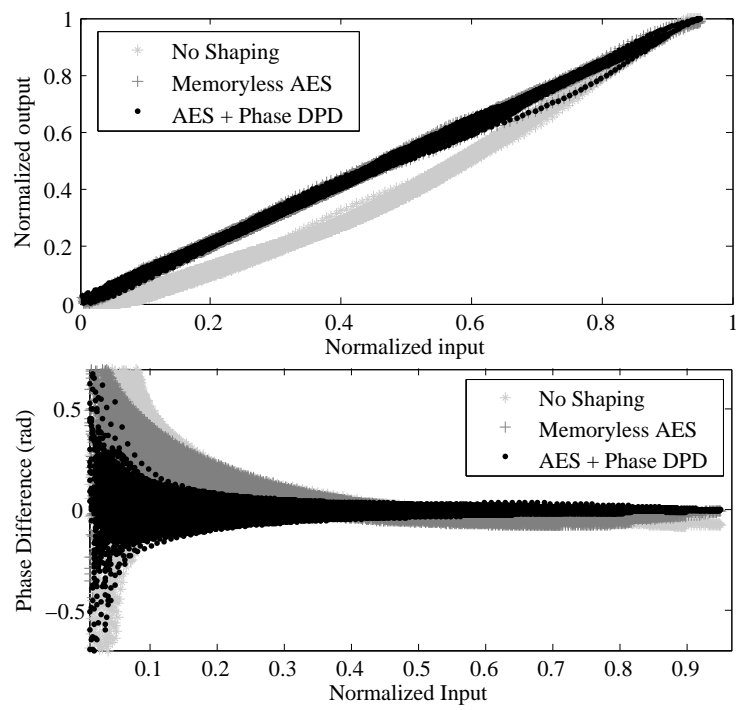

Fig. 3. RF PA AM-AM and AM-PM characteristics under ET mode.

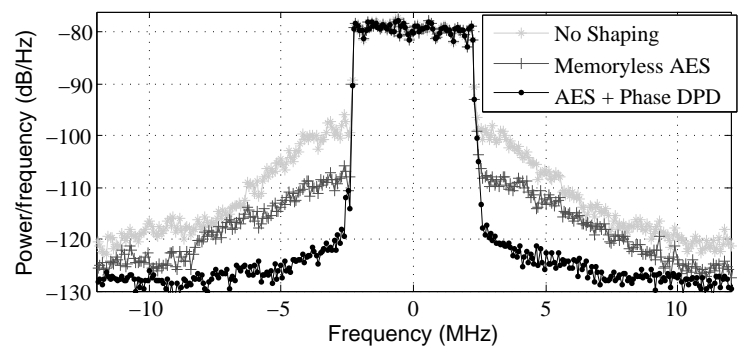

Fig. 4. RF PA output power spectra under ET mode.

mode, the AM-PM distortion results less harmful (except for the feedthrough effect at low drain voltage supply levels) and with only AES, in 3 iterations (at least 25 are required in ET mode) the linearization specifications were met. For handsets or low/medium power equipment, the PA has to be carefully designed to show a linear AM-PM characteristic and thus avoiding additional DPD compensation in the I-Q path.

\section{REFERENCES}

[1] R. Wu, Y.-T. Liu, J. Lopez, C. Schecht, Y. Li, and D. Lie, "High-Efficiency Silicon-Based Envelope-Tracking Power Amplifier Design With Envelope
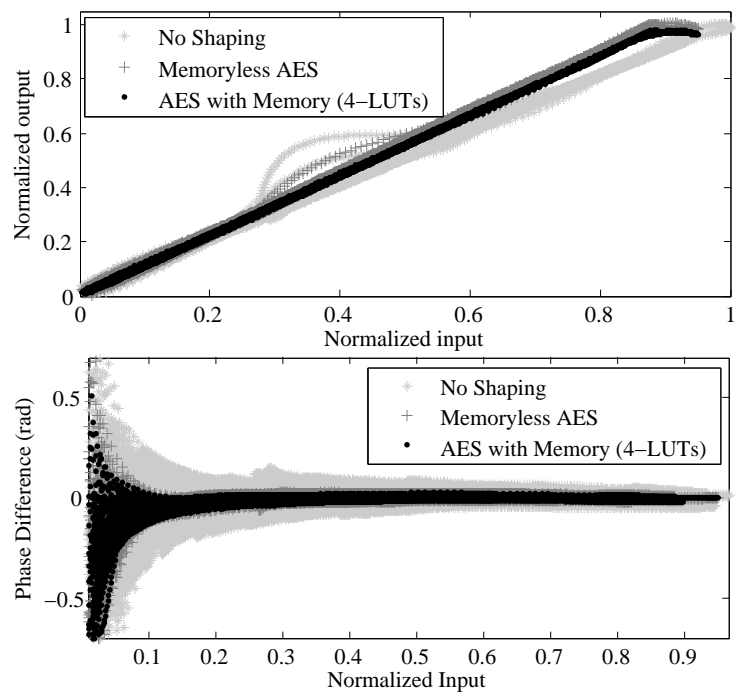

Fig. 5. RF PA AM-AM and AM-PM characteristics under EER mode.

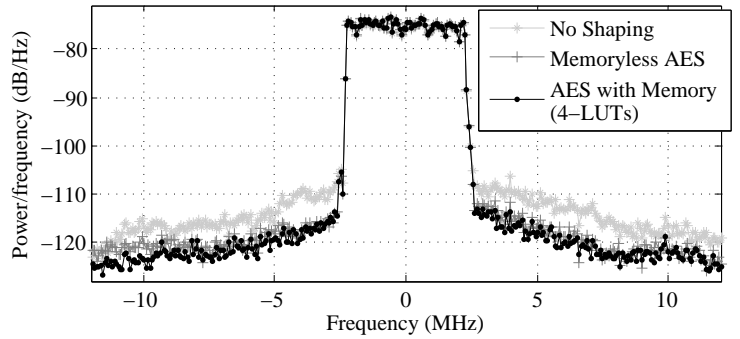

Fig. 6. RF PA output power spectra under EER mode.

Shaping for Broadband Wireless Applications," IEEE Journal of SolidState Circuits, vol. 48, no. 9, pp. 2030-2040, Sept 2013.

[2] N. Giovannelli, T. Vlasits, A. Cidronali, and G. Manes, "Efficiency and linearity enhancements with envelope shaping control in wideband envelope tracking GaAs PA," in Workshop on Integrated Nonlinear Microw. and Millimetre-Wave Circuits (INMMIC), April 2011, pp. 1-4.

[3] T. Rautio, H. Harju, S. Hietakangas, and T. Rahkonen, "Envelope tracking power amplifier with static predistortion linearization," Int. Journal of Circuit Theory and Applications, vol. 37, no. 2, pp. 365-375, 2009.

[4] J. C. Pedro, L. C. Nunes, and P. M. Cabral, "Soft compression and the origins of nonlinear behavior of GaN HEMTs," in 44th European Microwave Conference (EuMC), Oct 2014, pp. 1297-1300.

[5] P. L. Gilabert and G. Montoro, "3-D Distributed Memory Polynomial Behavioral Model for Concurrent Dual-Band ET PA Linearization," IEEE Trans. on Microw. Theory and Tech., vol. 63, pp. 638-648, Feb 2015. 